\title{
Estudios sobre el desarrollo económico español, dedicados al profesor Eloy Fernández Clemente
}

\author{
Título: Estudios sobre el desarrollo económico español, \\ dedicados al profesor Eloy Fernández Clemente. \\ Autores: Domingo Gallego, Luis Germán y Vicente Pinilla (eds.) \\ Edición: Zaragoza, Prensas de la Universidad de Zaragoza, 2016, 408 pp. \\ ISBN. 978-84-16515-38-7 \\ Ricardo ROBLEDO \\ Universidad de Salamanca y Universitat Pompeu Fabra
}

\begin{abstract}
"Este no es un libro de afectos". Así inició el coordinador la presentación del libro homenaje que diversos profesores de Aragón tributaron hace poco tiempo a Eloy Fernández Clemente, como un ejercicio de memoria individual y colectiva (Rújula, 2010). El libro que presento huye aún más si cabe de aquella declaración, lo cual no quiere decir que se huya del afecto, como testimonian las dedicatorias de los autores, sino que se canaliza en un libro articulado sobre el desarrollo económico español desde una perspectiva macro. No se trata pues de una yuxtaposición de colaboraciones, lo que no evita que haya diferentes enfoques sobre un mismo tema, fruto de estrategias de investigación distintas. Eso da lugar a contrastes y complementariedades.

Como señalan Salvador Almenar y Alfonso Sánchez Hormigo al final de su capítulo no son pocos los trabajos de Eloy Fernández Clemente dedicados a la historia del pensamiento económico: "No solo se trata de la Ilustración o Joaquín Costa, dos inmensos filones que han recibido su atención permanente, sino de un conjunto de estudios sobre los aragoneses que fueron ministros de Hacienda (desde Garay a Navarro Rubio), otro conjunto dedicado a economistas del XIX (Rodríguez, Carreras, Piernas) y un grupo que estudia y recupera los economistas del exilio republicano español (Sánchez Sarto, Viñuales)" (p. 60).

He preferido agrupar los capítulos en torno a cinco temas aunque para ello no siga a veces el orden con que aparecen en el libro.
\end{abstract}

\section{Historia, teoría, política}

Este es un libro en el que se dialoga, aunque sea de forma implícita o indirecta, sobre la relación entre historia y teoría. Se trata de un diálogo que en otros casos se ha resuelto con desplantes o descalificaciones. En el libro Estudios sobre el desarrollo del económico español (título que evoca en cierto modo la obra de M. Dobb) el diálogo es creativo pues no hay perspectivas unidimensionales. Según sea el tema se acude a la historia medioambiental, a la nueva geografía económica y el desarrollo endógeno, a la historia antropométrica, entre otras perspectivas. Y se reivindica el matrimonio conveniente entre historia de las ideas y de la teoría como exponen los profesores Almenar y Sánchez Hormigo en el capítulo ["En pos de la prosperidad. Un esquema sobre las percepciones. Del atraso y el desarrollo de los primeros economistas clásicos españoles (1790-1850)"]. En su texto se pasa revista a una serie de trabajos de Flórez Estrada, Jaumeandreu, Colmeiro, entre otros, influidos por los principales pensadores de la época (Smith, Ricardo, Say, Sismondi) y por las realizaciones y prácticas políticas de los países vecinos. Las reflexiones y soluciones que se presentan son variadas, pero, eso sí, tienden a coincidir en la importancia del desarrollo industrial.

Almenar y Sánchez Hormigo inician la exposición con la ingeniosa pregunta de Blaug «¿Historia de las ideas? No, gracias, somos economistas», frase que sanciona la inferioridad de condiciones en que se halla la disciplina en muchas facultades de economía. En estos primeros párrafos se llama la atención sobre los peligros de una formación alicorta, en la que se ignora todo menos el presente. Como apuntan ellos, solo muy recientemente Dani Rodrik (2014) ha destacado que las «ideas están extrañamente ausentes de los actuales modelos de economía política», aun cuando las ideas intervengan realmente en la formación de las preferencias, restricciones y variables sujetas a elección en los modelos de elección racional. 
Ejemplo adicional de que este libro no es prisionero de un concepto estrecho del desarrollo es que hay historia cuantitativa en varios capítulos, pero los datos se utilizan de forma inteligente sin forzar razonamientos cuantitativistas. Al hacer esto, se ha seguido una tradición venerable, desde Adam Smith, no muy entusiasta con la econometría del momento, que no era otra que la aritmética política de William Petty, hasta Keynes que en el Tratado de Probabilidad mostró sus preferencias por los datos crudos, sin mucha cocina "para que la observación atenta de la realidad cambiante no se viera dificultada por engendros y manipulaciones..." (Skidelsky, 2013, p. 410).

Quisiera resaltar también la complejidad del libro por la incorporación de variables como la política. Josep Fontana dedica el primer capítulo ("El fin del crecimiento. Sobre el uso político de la historia económica"), a cuestionar el relato dominante de un proceso ininterrumpido del progreso. Al comentar el tema de la desigualdad, tiene ocasión de criticar la interpretación de Piketty (El capital en el siglo XXI) por no tomar en consideración las circunstancias políticas globales, tales como la guerra fría y el papel representado en esta etapa por la actuación de los sindicatos, para explicar la época de prosperidad global y de aumento de los niveles de vida que se produjo entre 1945 y 1975. Igualmente hace notar que en la fase siguiente el crecimiento de la desigualdad no se explicaría correctamente sin el peligro de la amenaza soviética y la política de lucha contra los sindicatos.

Es también la variable política a la que acude F. Comín en el capítulo ("Industrialización y desarrollo económico en la España contemporánea: una perspectiva histórica") para quitar importancia a las explicaciones hacendísticas o al deficiente papel del Estado en el fracaso de la industrialización. Estaríamos ante una explicación fallida "porque, al fin al cabo, el Estado no deja de ser una "ficción legal"; es decir una institución ocupada por políticos, con nombres y apellidos, cuyas actuaciones se guían por las acciones de lobby de los grupos de presión (el regulador cae cautivo del regulado casi siempre), generalmente empresariales, también con intereses personales y societarios concretos" (p. 64). Y esto ha sucedido con sufragio censitario, dictaduras del siglo XX o democracia porque con independencia de los resultados en las urnas, la política económica de los gobiernos tiende a respetar y a favorecer a los grupos de presión consolidados.

\section{Crecimiento económico ¿una perspectiva sombría?}

$\mathrm{Al}$ igual que ocurrió a fines del XIX con la visión pesimista asociada al "climaterio británico" que nos dejó Arnold Toynbee (1852-1883), difusor del término "revolución industrial" (Toynbee, 1884), también en este libro hay colaboraciones que transmiten perspectivas poco complacientes sobre el desarrollo económico en general y sobre el español en particular. En el texto de Fontana se recoge la literatura que ha empezado a plantear dudas, especialmente a partir de 2012, sobre la posibilidad del modelo de crecimiento continuado. Resulta interesante que se haya recuperado ahora el discurso de Alvin Hansen de 1938 (Hansen, 1939) en la American Economic Association sobre el estancamiento secular y las "débiles y anémicas recuperaciones" que dificultaban el pleno empleo (p. 31).

Por lo que se refiere a España, además del capítulo de Comín donde se demuestra sistemáticamente que España nunca ha culminado el proceso de industrialización, las páginas que más recortan el horizonte de un progreso constante son las de I. Iriarte y E. Tello ("El crecimiento económico moderno en España en perspectiva ambiental: un estado de la cuestión"). Si se acepta esta perspectiva es comprensible que se juzgue contradictorio el paradigma que asume, implícita o explícitamente, la posibilidad de un crecimiento infinito ignorando que el mismo se sustenta y despliega en una biosfera finita. Las herramientas que utilizan estos autores son las de la huella ecológica y los balances energéticos, pero acuden también al análisis institucional para defender que en España como en el resto del mundo el crecimiento económico ha comportado el establecimiento de nuevas relaciones entre sociedad, economía y naturaleza (p. 118). De los múltiples desequilibrios de que dan cuenta en el capítulo 4, señalo dos caras de una misma moneda. A principios del siglo XXI, "el litoral mediterráneo y la conurbación madrileña concentraban casi un $40 \%$ de la población total, pese a representar en torno al $8 \%$ del territorio. La cifra alcanza hasta el $63 \%$ si atendemos solo a la población urbana" (p. 101). Por la otra parte, la dinámica de la revolución verde obliga a concentrar la actividad agraria directa en superficies 
reducidas de uso intensivo, mientras se desaprovechan amplísimos espacios que antes se usaban para proveer de nutrientes a las áreas roturadas. En suma, Iriarte y Tello defienden el papel de la historia económica y ambiental para analizar las sendas del crecimiento del pasado y detectar los problemas que ha generado sin dejarse deslumbrar por el espejismo de verlo únicamente como un éxito (p. 119).

Diferente perspectiva es la que emplea Alfonso Herranz ("Una aproximación a la integración de los mercados españoles durante el siglo XIX"). Al centrarse en un periodo en el que las economías orgánicas eran las dominantes, es natural que no se contemple en su estudio esta deriva de fuertes desequilibrios territoriales. Una parte del trabajo consiste en analizar la historiografía española sobre la integración de mercados, distinguiendo entre integración de mercados, requisito imprescindible de la integración económica. La otra parte se centra en la cronología de la integración de mercados y recuerda una vieja polémica que en los años 80 del siglo pasado enfrentó a partidarios y detractores del papel de ferrocarril en la integración del mercado interior. Herranz sitúa al final de la década ominosa una mejora sustancial de las comunicaciones mediante la inversión en carreteras, mejoras sustanciales en la organización del transporte regular de viajeros y en la información, aunque siempre sobre la base de las tecnologías de transporte tradicionales. Esta fue también la experiencia europea. Se enlaza así con la influyente tesis de Madrazo y otros autores que percibieron cambios sustanciales en la articulación del mercado interior antes del desarrollo de la red ferroviaria.

\section{La doble cara de la agricultura}

Hay dos capítulos, además del de Iriarte y Tello, que testimonian bien la importancia que el departamento de historia económica de Zaragoza fundado por Eloy Fernández Clemente ha concedido a la historia agraria. En páginas anteriores ya han aparecido varias referencias al respecto, por ejemplo, a la desagrarización y despoblación rural que tienen en Aragón uno de los mejores escenarios. Se trata de dos capítulos que no se solapan lo más mínimo. El primero de Ernesto Clar, Miguel Martín-Retortillo y Vicente Pinilla (“Agricultura y desarrollo económico en España, 1870-2000") expone el análisis del cambio agrario en el largo plazo, mientras que el de Domingo Gallego ("Agricultura, desarrollo económico y democracia") pretende utilizar la experiencia del pasado que pueda ser útil para los países ruralizados y pobres de hoy.

El primer capítulo participa de la literatura "revisionista" sobre el papel central que solía concedérsele a la agricultura en el crecimiento económico. En vez de considerarla responsable del atraso, los autores prefieren hablar del "bajo desarrollo de las actividades que debían haber ejercido el liderazgo, generando así un bajo nivel de demanda urbana de productos agrarios, que tenían, además, un carácter bastante tradicional" (p. 182). En el capítulo se hace referencia a las restricciones medioambientales o institucionales si bien el núcleo de la argumentación para explicar el bajo consumo de abonos, la tardía mecanización o un incremento más lento de la productividad antes de 1940 se concreta principalmente en el precio de los factores productivos que no incentivaban el cambio en la dirección adecuada. Después de la vuelta al pasado y la tímida rectificación de 1940-1959 (rectificación forzada por las quejas de los trabajadores urbanos más que de los agricultores), se llega a la etapa de la modernización acelerada y de la integración europea (1960-2000) en la que tierra y trabajo perdieron protagonismo en el proceso productivo en favor del capital: esto aceleró la implantación de la ganadería intensiva industrial, las exportaciones agrarias o el desarrollo del complejo agroalimentario. Hay otros aspectos que se escapan inevitablemente en estas visiones de síntesis. Al final el sector agrario tiene un papel cada vez menor en el crecimiento económico mientras recupera protagonismo en el desarrollo económico, mediante la conservación de la naturaleza y la lucha contra la despoblación. En mi opinión los autores se inclinan por una solución de cierto compromiso entre viejas y nuevas tesis cuando afirman que el sector agrario estuvo caracterizado por dos tendencias contrapuestas: no fue suficientemente dinámico para ser el impulsor clave del proceso de industrialización; pero no fue tan estático como para ser el factor que explique el lento ritmo de la industrialización española.

Gracias al recurso de la economía evolutiva y la teoría del metabolismo social Domingo Gallego defiende en su capítulo una lógica distinta a la de la inevitabilidad de la revolución verde. La historia económica tiene que cambiar de referentes: no hay por qué seguir el modelo prefija- 
do de la vía histórica inglesa del crecimiento económico que llevó a la descampesinización a principios del siglo XIX. El objetivo de este ensayo es resaltar los peligros de las políticas desruralizadoras cuando las sociedades cuya prosperidad se quiere impulsar son pobres y ruralizadas. El enfoque se aparta, por tanto, de la visión convencional del crecimiento económico indefinido, ya criticado en el apartado anterior, y obliga a mirar el pasado de las sociedades agrarias españolas con otras miradas más comprensivas y relativamente optimistas. Si se apuesta por un crecimiento económico integrador habrá que tener en cuenta las capacidades productivas que permitieron la gestión del medio natural igual que el desarrollo de múltiples actividades económicas no agrarias. También aborda Gallego "la persistencia de sus capacidades organizativas familiares, interfamiliares, comunales, cooperativas, sindicales y empresariales. Todas estas capacidades serían vitales tanto para organizar procesos productivos agrarios y no agrarios como para constituir en las áreas rurales grupos capaces de autogobernarse de forma abierta y de ser interlocutores activos en el diseño y aplicación de la política agraria y económica en general" (p. 15). En vez de considerar al campesinado una mercancía devaluada que es triturada en el basurero de la historia, el fomento de lo rural se plantea como una oportunidad para favorecer la construcción de sociedades menos agresivas ambientalmente y menos desequilibradas socialmente.

\section{Desigualdad del crecimiento}

Los capítulos que se resumen en este epígrafe forman parte de la segunda parte del libro que los editores han agrupado bajo el epígrafe "Consecuencias del crecimiento económico" y que este reseñante expone desde el ángulo de la desigualdad que afecta a los espacios, a las características físicas de las personas, a los modelos nutricionales o a la seguridad laboral.

En el capítulo de Luis Germán ("Mosaico hispánico. Crecimiento, especialización productiva y desigualdades regionales en la España Contemporánea") se analizan las fuerzas que conducen a la centralización espacial de la actividad económica utilizando la línea argumental de la nueva geografía económica. A lo largo de esta exposición se muestra cómo el crecimiento económico moderno español - inductor del cambio estructural - es un proceso desequilibrador, que generó polarización. Esta se acentuó especialmente en el contexto de la implantación del nuevo Estado liberal y de la consecución de su prioritario reto económico, la formación del mercado nacional. A pesar del proceso de convergencia habido durante el tercer cuarto del siglo XX protagonizado esencialmente por las migraciones internas - las regiones agrarias se mantenían a finales de siglo en los últimos lugares del ranking regional (menores niveles en PIB pc, productividad, tasa actividad, I + D, infraestructuras, tasa de emprendimiento...).

Si en este capítulo se comprueba cómo Andalucía -que contaba a mediados del siglo XIX con una especialización agraria e industrial (y superior nivel de PIB pc)- perdió esta con el nuevo siglo XX y pasó a contar solo con especialización agraria, inicialmente poco eficiente, perdiendo asimismo en la segunda mitad del novecientos su superior orientación exportadora, en el siguiente capítulo Martínez Carrión ("Variaciones provinciales del estado nutricional de los españoles durante la Restauración") certifica cómo a mediados del siglo XX Andalucía figura ya entre las regiones con tallas más bajas acorde con su pobres niveles de renta y desarrollo económico. No había sido así antes pues según los datos antropométricos de los soldados españoles nacidos en 1883-1886, recopilados por Sánchez Fernández en 1913, los territorios de la periferia incluyendo en ella a Asturias y gran parte de Andalucía exhibían tallas por encima del promedio español cifrado en 163,6 cm. La importancia de la malnutrición moderada en las diferencias interprovinciales de la talla se ve reforzada por el peso e índice de la masa corporal de los reclutas útiles de 1903-1906. Por un lado la España húmeda, la España mediterránea (hasta Alicante) y el valle del Ebro destacan por sus relativas altas tallas, pesos e índices de masa corporal frente a lo que sucedía en buena parte de las regiones de la España interior.

De la desigualdad en el peso o en el índice de la masa corporal se pasa a la desigualdad del consumo alimentario en el capítulo de F. Collantes ("A la mesa con Malassis. Modelos de consumo alimentario en la España contemporánea") en el que se traza una panorámica sobre la evolución de la alimentación de la población española a lo largo del último siglo y medio utilizando el concepto de "modelo de consumo alimentario" de Malassis en vez del de "transición nutricional" que es el que han seguido otros autores. Collantes propone, en concreto, que a lo 
largo del periodo contemporáneo se sucedieron en España cuatro modelos de consumo alimentario: un primer modelo hasta finales del siglo XIX, en el que domina la precariedad generalizada de unas dietas de base vegetal y orgánica; un segundo modelo entre 1880/1900 y 1950/1960, caracterizado por un crecimiento de las ingestas y un desarrollo embrionario de cambios en la composición y segmentación de los consumos; un tercer modelo entre 1950/1960 y 1980/1990, en el que se produce el paso a un consumo alimentario masivo, cada vez más abundante y más generalizado entre todos los estratos sociales, y con un peso cada vez mayor para los alimentos de origen animal y los alimentos procesados; y finalmente, un cuarto régimen a partir de 1980/1990 en el que entran en juego, entre otras cosas, las "sustituciones cualitativas" de Malassis entre alternativas alimentarias cada vez más diversificadas y con un elevado grado de complejidad en su proceso de producción y comercialización.

Javier Silvestre ["Industrialización y accidentes de trabajo en España (finales del siglo XIX y primer tercio del siglo XX")] inicia su capítulo con la afirmación de que "La Revolución industrial probablemente aumentó el número de muertos y heridos en el trabajo", situación que a medio-largo plazo tuvo que mejorar por el cambio tecnológico, las mejoras sociales y los inicios de la regulación del mercado de trabajo (seguros de accidentes e inspectores de trabajo). Dicha regulación se retrasó hasta fines del siglo XIX y en mi opinión no fue dádiva de un estado benefactor sino exigencia del movimiento obrero organizado. La acción pública en lo que se refiere a la legislación y a la inspección en España fue tardía, y los criterios de gestión de la inspección fueron más de tipo «persuasivo que coercitivo». Los seguros y los organismos promotores de la seguridad tuvieron menos desarrollo en España y ello favoreció el menor esfuerzo en prevención. Según Silvestre, con las cautelas que imponen las estadísticas, "parece ser que la seguridad empeoró conforme avanzó el primer tercio del siglo XX" (p. 308). Se puede afirmar que las externalidades negativas vía accidentes inducidas por la industrialización fueron en menor medida corregidas por la acción pública, privada y mercantil en España que en otros países. Si pese a ello la intensidad de accidentes fue similar, quizá se debiese al menor desarrollo del proceso de industrialización.

Antonio M. Bernal cierra la segunda parte del libro [("La Cámara de Comercio de Sevilla en la coyuntura finisecular (1886-1912)]. En España las Cámaras aparecen aupadas por el movimiento proclive al libre comercio y fueron una creación de la política reformista del gobierno de Sagasta en 1886, pensadas más para la eficiencia de la Administración del Estado que para la transformación del tejido económico y social (p. 336). La Cámara daba cobijo a los empresarios integrados en distintos ámbitos de la economía sevillana movilizados por la política arancelaria y tratados de comercio o la gestión de Cuba y sus consecuencias. La crisis de 1898 derivada de la guerra contra los Estados Unidos de América actuó como un activo revulsivo de la vida cameral. Al igual que otras cámaras, la de Sevilla funcionaba como centro de formación de opinión para la movilización de unos intereses que podían entrar en conflicto con los de otras cámaras que tenían una orientación productiva distinta.

\section{Desarrollo de la nación imperial}

El funcionamiento de la nación imperial se asentó en la necesidad de leyes especiales para las colonias que Napoleón puso en funcionamiento después del 18 Brumario, un principio de discrecionalidad de actuación que adoptarían las naciones imperiales del siglo XIX. Es ese principio de discrecionalidad el que permitió a Napoleón restablecer la esclavitud en 1794 estando prohibida en la Francia europea. Funcionaron así las dobles constituciones (Fradera, 2015, XII, p. 1108). Aunque hubo que hacer reacomodos como ocurrió en Cuba después de 1868, eso no ocurrió en Filipinas, convertida en una colonia pura y dura, situación muy condicionada por la demografía -siete a ocho mil españoles frente a tres millones de nativos- en un espacio tan disperso como el archipiélago filipino. Estas circunstancias no permitían compensaciones como las que se conseguían en las Antillas.

Este es el contexto en el que se integra el último capítulo de Luis Alonso ("Las haciendas de las órdenes regulares en las Islas Filipinas. Los intereses de los frailes predicadores, 15871800") que abarca algo más que las Islas al estar presente México, estudio comparativo obligado pues en la conquista de las Islas participaron los hispanoamericanos trasladando las prácticas y las estructuras sociales de Nueva España. Aunque la iglesia estaba excluida de la propiedad de 
la tierra, L. Alonso demuestra las formas de acceso que consiguieron las órdenes religiosas por el mecanismo de las ocupaciones, ocupaciones que eran ilegales, pero como si fueran un pecado, se regularizaban con una penitencia o una multa. Como ocurrió en España con los comunales, tal situación no fue sancionada con la pasividad de los afectados. Las comunidades de indígenas se rebelaron pero también el regalismo vino en ayuda de la Hacienda para reclamar su parte de la tarta. De la economía de las haciendas dominicanas destaco el papel tan importante que desempeñaron los grandes colonos, similar al de esa clase intermedia tan bien estudiada en Salamanca o en Extremadura: consiguieron acumular beneficios sobre todo con la exportación de azúcar y mandar a sus hijos a estudiar en la metrópoli como hizo Rizal. Pero cuando los dominicos comprobaron que los beneficios provenían del subarriendo, recuperaron la explotación directa y echaron a los colonos, entre ellos a la familia de Francico Rizal en 1878. Años después, su hijo el doctor José Rizal se convirtió en el padre de la emancipación filipina.

Considero un buen final que un libro sobre el desarrollo económico español centrado en los dos últimos siglos lo haga con un estudio sobre la economía colonial, allí donde funcionaba la otra constitución. Sin el recurso de las colonias se harían incomprensibles los destinos de la exportación de harinas y otros productos, la formación de capitales e incluso la preferencia de nuestros emigrantes (Robledo, 2010).

\section{Bibliografía}

Fradera, Josep Maria. 2015. La nación imperial (1750-1918). Barcelona, Edhasa.

Hansen, Alvin. 1939. Economic Progress and Declining population Growth, American Economic Review, XXIX (1), pp. 1-15.

Langreo, Alicia, García Álvarez-Coque, José María. 2011. El Ministerio de Agricultura, Pesca y Alimentación, 1986-2007. En R. Robledo (coord.), Historia del Ministerio de Agricultura, 1900-2008. Madrid, Ministerio de Medio Ambiente, Medio Rural y Marino, 291-325.

Rodrick, Danni. 2014. When Ideas Trump Interests: Preferences, Worldviews, and Policy Innovations, Journal of Economic Perspectives vol. 28 (1), Winter, 189-208.

Robledo, Ricardo. 2010. "Todo era América": despoblación rural y políticas de inmigración (1850-1930), Mundo Agrario, núm. 20 (on line).

Rújula, Pedro (coord.). 2011. Eloy Fernández Clemente. El tiempo y la historia. Zaragoza, Ayuntamiento de Andorra y Centro de Estudios Locales de Andorra.

Skidelsky, Robert. 2014. John Maynard Keynes. Barcelona, Península.

Toynbee, Arnold. 1884. Lectures on the Industrial Revolution in England: Public Addresses, Notes and Other Fragments, together with a Short Memoir by B. Jowett. London, Rivington's. 